\title{
Article
}

\section{The Proof of a Conjecture Relating Catalan Numbers to an Averaged Mandelbrot-Möbius Iterated Function}

\author{
Pavel Trojovský *D and K Venkatachalam
}

check for updates

Citation: Trojovský, P.;

Venkatachalam, K. The Proof of a Conjecture Relating Catalan Numbers to an Averaged Mandelbrot-Möbius Iterated Function. Fractal Fract. 2021, 5, 92. https://doi.org/

10.3390 / fractalfract5030092

Academic Editor: Michel L. Lapidus

Received: 17 July 2021

Accepted: 9 August 2021

Published: 11 August 2021

Publisher's Note: MDPI stays neutral with regard to jurisdictional claims in published maps and institutional affiliations.

Copyright: (C) 2021 by the authors. Licensee MDPI, Basel, Switzerland. This article is an open access article distributed under the terms and conditions of the Creative Commons Attribution (CC BY) license (https:/ / creativecommons.org/licenses/by/ $4.0 /)$.
Department of Mathematics, Faculty of Science, University of Hradec Králové, 50003 Hradec Králové, Czech Republic; venkatachalam.k@christuniversity.in

* Correspondence: pavel.trojovsky@uhk.cz; Tel.: +42-049-333-2860

\begin{abstract}
In 2021, Mork and Ulness studied the Mandelbrot and Julia sets for a generalization of the well-explored function $\eta_{\lambda}(z)=z^{2}+\lambda$. Their generalization was based on the composition of $\eta_{\lambda}$ with the Möbius transformation $\mu(z)=\frac{1}{z}$ at each iteration step. Furthermore, they posed a conjecture providing a relation between the coefficients of (each order) iterated series of $\mu\left(\eta_{\lambda}(z)\right)$ (at $\left.z=0\right)$ and the Catalan numbers. In this paper, in particular, we prove this conjecture in a more precise (quantitative) formulation.
\end{abstract}

Keywords: fractal; Mandelbrot set; Julia set; Möbius transformation; iterated function; Catalan numbers

\section{Introduction}

Let $\eta: \mathbb{C} \rightarrow \mathbb{C}$ be a monic complex polynomial of degree $d \geq 2$. We denote by $\eta^{j}$ the $j$-th iterate of $\eta$, that is,

$$
\eta^{j}(z)=\overbrace{\eta(\eta(\eta(\cdots \eta}^{j \text {-times }}(z) \cdots))) .
$$

The filled-in Julia set of $\eta$ is defined as

$$
K(\eta)=\left\{z \in \mathbb{C}: \eta^{j}(z) \text { does not diverge }\right\}
$$

and the Julia set $J(\eta)$ of the function $\eta$ is defined to be the boundary of the set $K(\eta)$, i.e., $J(\eta)=\partial K(\eta)$ (see, e.g., [1]).

In this work, we are interested in a modified version of the "classical" filled-in Julia set $K\left(\eta_{\lambda}\right)$ and the Julia set $J\left(\eta_{\lambda}\right)$ of functions in the quadratic family $\left(\eta_{\lambda}(z)\right)_{\lambda \in \mathbb{C}}=\left(z^{2}+\lambda\right)_{\lambda \in \mathbb{C}}$. We observe that the Mandelbrot set $M\left(\eta_{\lambda}\right)$ is the fractal defined as

$$
M\left(\eta_{\lambda}\right)=\left\{\lambda \in \mathbb{C}: J\left(\eta_{\lambda}\right) \text { is connected }\right\} .
$$

We point out that there is a more "workable" way of considering the Mandelbrot set (we refer to [2], Theorem 14.14) for a proof of the usually referred fundamental theorem of the Mandelbrot set):

$$
\lambda \in M\left(\eta_{\lambda}\right) \Longleftrightarrow \eta_{\lambda}^{j}(0) \text { does not diverge } .
$$

Some other recent results related to the Mandelbrot set can be found for example in [3-10].

In 2019, Mork et al. [11] constructed filled-in Julia sets for a lacunary function $\eta_{N, k}(z)=$ $\sum_{n=1}^{N} z^{P_{k}(n)}$, where $\left(P_{n}(k)\right)_{n}=\left(\frac{1}{2}\left(k n^{2}-k n-2\right)\right)_{n}$ is the sequence of centered $k$-gonal numbers and $k$ is any positive integer (for more facts and history of lacunary functions see, e.g., [12-14]).

In 2021, Mork et al. [15] followed up on the aforementioned article and considered a generalization of the filled-in Julia sets and their corresponding Mandelbrot sets by 
composing the lacunary function $\eta(z)=\sum_{n=1}^{N} z^{P_{k}(n)}$ with a fixed Möbius transformation $\mathcal{M}(z)=e^{i \theta} \frac{z-a}{\bar{a} z-1}$ (with $(\theta, a) \in \mathbb{R} \times \mathbb{D}$, where $\mathbb{D}$ denotes the the unit disc) at each iteration step. More precisely

$$
h^{j}(z ; a, k, N, \theta)=\overbrace{\mathcal{M}\left(\eta _ { N , k } \left(\mathcal { M } \left(\eta _ { N , k } \left(\cdots \mathcal { M } \left(\eta_{N, k}\right.\right.\right.\right.\right.}^{j-\text { times }}(z) \cdots))))) .
$$

Very recently, Mork and Ulness [16] continued the previous line of research by dealing with the so-called $j$-averaged Mandelbrot set which is a set generated by iterating a function obtained by composing the function $\eta_{\lambda}$ and the Möbius transformation $\mu_{A}(z)=\frac{a z+b}{c z+d}$, where $A=(a, b, c, d) \in \mathbb{C}^{4}$. Thus,

$$
h^{j}(z ; A)=\overbrace{\mu_{A}\left(\eta _ { \lambda } \left(\mu _ { A } \left(\eta _ { \lambda } \left(\cdots \mu _ { A } \left(\eta_{\lambda}\right.\right.\right.\right.\right.}^{j \text {-times }}(z) \cdots))))) .
$$

The name " $j$-averaged" is used here since the points of the resulting fractal are colored according to the total number of members of the following sequence of iterations $\left(\mathcal{H}_{n}\right)_{0 \leq n \leq j}$, that escaped from the circle with radius 2 (the concrete algorithm for coloring of points of this fractal you can find in Appendix 1 of [16]), see Figure 1,

$$
\begin{aligned}
\left(\mathcal{H}_{n}\right)_{0 \leq n \leq j} & =\left\{h^{0}(0 ; A), h^{1}(0 ; A), \ldots, h^{j}(0 ; A)\right\} \\
& \left.\left.\left.\left.\left.=\{0, \mu_{A}\left(\eta_{\lambda}(0)\right), \ldots, \overbrace{\mu_{A}\left(\eta _ { \lambda } \left(\mu _ { A } \left(\eta _ { \lambda } \left(\cdots \mu _ { A } \left(\eta_{\lambda}\right.\right.\right.\right.\right.}^{j}(0) \cdots)\right)\right)\right)\right)\right\} .
\end{aligned}
$$
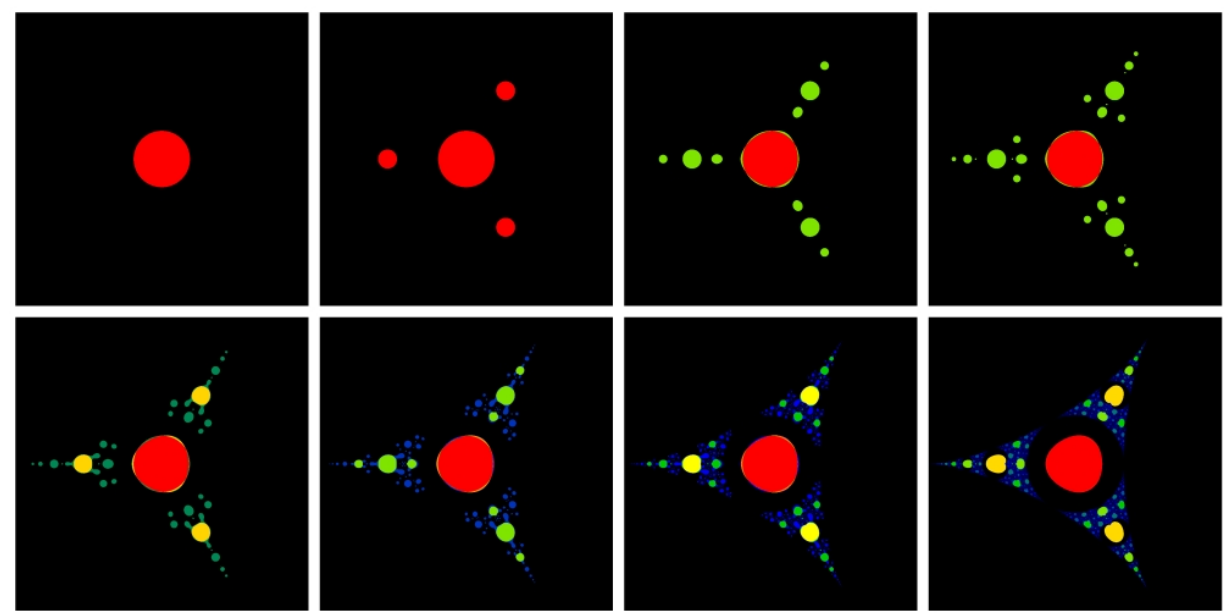

Figure 1. The $j$-averaged Mandelbrot sets for $A=(0,0.5,1,0), \lambda=x+i y$, with $x \in[-1.2,0.8]$, $y \in[-1,1], j=1,2,3,4$ (the first row from the left to the right) and for and $j=5,7,10,100$ (the second row from the left to the right). We used functions in the software Mathematica ${ }^{\circledR}$ (see [17]) that are defined in Appendix 1 of [16].

Mork and Ulness ([16] Theorem 1) proved that the $j$-averaged Mandelbrot set for the Möbius transformation $\mu_{A}$ with $A=(0,1,1,0)$ has threefold rotational symmetry and dihedral mirror symmetry. Additionally, they raised a conjecture (see [16], Conjecture 2)) concerning the coefficients of these iterations. Before stating their conjecture, we introduce some basic notations.

Let $\lambda \in \mathbb{D}$ be a non-zero complex number. Define the function $H(z, \lambda)$ by $H(z, \lambda):=$ $\mu_{A}\left(\eta_{\lambda}(z)\right)$, with $A=(0,1,1,0)$. Therefore,

$$
H(z, \lambda)=\frac{1}{z^{2}+\lambda} .
$$


Observe that the $n$-th iteration of $H$ at $z=0$ is a function of $\lambda$, say $h_{n}(\lambda)$, which satisfies the relations:

$$
h_{0}(\lambda)=0, h_{1}(\lambda)=H(0, \lambda)=\frac{1}{\lambda} \quad \text { and } \quad h_{n+1}(\lambda)=H\left(h_{n}(\lambda), \lambda\right), \text { for } n \geq 1 .
$$

The sequence $\left(C_{n}\right)_{n \geq 0}$ of the Catalan numbers, which is called the sequence A000108 in the OEIS [18], is often defined with the help of the central binomial coefficient $\left(\begin{array}{c}2 n \\ n\end{array}\right)$ by

$$
C_{n}=\frac{1}{n+1}\left(\begin{array}{c}
2 n \\
n
\end{array}\right)
$$

thus, its first terms are in Table 1.

Table 1. Values of $C_{n}$ for $n$ from 0 to 14 .

\begin{tabular}{cccccccccccccccc}
\hline$n$ & 0 & 1 & 2 & 3 & 4 & 5 & 6 & 7 & 8 & 9 & 10 & 11 & 12 & 13 & 14 \\
\hline$C_{n}$ & 1 & 1 & 2 & 5 & 14 & 42 & 132 & 429 & 1430 & 4862 & 16,796 & 58,786 & 208,012 & 742,900 & $2,674,440$ \\
\hline
\end{tabular}

which can lead us to the following recurrence relation (it was first discovered by Euler in 1761; for more facts, see [19])

$$
C_{n}=\frac{4 n-2}{n+1} C_{n-1}, \text { for } n \geq 1,
$$

with the initial condition $C_{0}=1$. Sometimes the sequence $\left(C_{n}\right)_{n \geq 0}$ is defined on the basis of the generating function $(1-\sqrt{1-4 x}) /(2 x)$, as the following holds (for $|x|<1 / 4$ )

$$
\sum_{n=0}^{\infty} \frac{\left(\begin{array}{c}
2 n \\
n
\end{array}\right)}{n+1} x^{n}=\frac{2}{1+\sqrt{1-4 x}}
$$

The aim of this paper is to obtain a (quantitative) result for the coefficients of the power series of $h_{n}(\lambda)$ which implies the Mork-Ulness' conjecture (qualitative version). More precisely,

Theorem 1. For all $n \geq 1$, we have

$$
h_{n}(\lambda)=\frac{1-(-1)^{n}}{2 \lambda}+(-1)^{n} \sum_{i=1}^{\lfloor n / 2\rfloor} C_{i-1} \lambda^{3 i-1}+O\left(\lambda^{3\lfloor n / 2\rfloor+2}\right),
$$

where $C_{n}$ is the $n$-th Catalan number.

Remark 1. We remark that Mork and Ulness [16] posed a slightly different conjecture. In fact, we can express their question by defining $h_{\infty}^{(1)}(\lambda)$ and $h_{\infty}^{(2)}(\lambda)$ as

$$
h_{\infty}^{(1)}(\lambda):=\lim _{n \rightarrow \infty} h_{2 n+1}(\lambda)=\frac{1}{\lambda}-\sum_{i \geq 0} C_{i} \lambda^{3 i+2}
$$

and

$$
h_{\infty}^{(2)}(\lambda):=\lim _{n \rightarrow \infty} h_{2 n}(\lambda)=\sum_{i \geq 0} C_{i} \lambda^{3 i+2} .
$$

They also asserted that these functions should converge in the whole unit disk (or the punctured one for $h_{\infty}^{(1)}(\lambda)$ ). However, this is not true (this is expected because of the exponential nature of Catalan numbers). For example, the simple bound $\left(\begin{array}{c}2 n \\ n\end{array}\right) \geq 4^{n} /(2 n+1)$, which comes from the fact that $4^{n}=(1+1)^{2 n}=\sum_{k=0}^{2 n}\left(\begin{array}{c}2 n \\ k\end{array}\right)$, implies that $C_{n}>4^{n} /\left(3 n^{2}\right)$ (some other bounds can be found in ([19] Chapter 2) and [20]) and so if $|\lambda|>1 / \sqrt[3]{2} \approx 0.793$ then $\left|C_{n} \lambda^{3 n+2}\right| \geq$ 
$3^{-1}|\lambda|^{2}\left(2^{n} / n^{2}\right)(\sqrt[3]{2}|\lambda|)^{3 n}>|\lambda|^{2} / 3$ (for $n \geq 4$ ) yielding the divergence of $h_{\infty}^{(2)}(\lambda)$. In order to compute the radius of convergence, say $r$, of $h_{\infty}^{(2)}(\lambda)$, one can write this function as $h_{\infty}^{(2)}(\lambda)=$ $\sum_{n \geq 0} a_{n} \lambda^{n}$, where

$$
a_{n}=\left\{\begin{array}{lll}
C_{(n-2) / 3}, & \text { if } n \equiv 2 \quad(\bmod 3), \\
0, & \text { if } n \neq \equiv 2 \quad(\bmod 3) .
\end{array}\right.
$$

Thus, $1 / r=\limsup _{n \rightarrow \infty} \sqrt[n]{a_{n}}$ and, by using $C_{n} \approx 4^{n} /\left(n^{3 / 2} \sqrt{\pi}\right)$ (which comes from the Stirling formula $\left.n ! \approx \sqrt{2 \pi n}(n / e)^{n}\right)$, we obtain

$$
\begin{aligned}
\frac{1}{r} & =\limsup _{n \rightarrow \infty} \sqrt[n]{a_{n}} \\
& =\limsup _{n \rightarrow \infty} \sqrt[3 n+2]{C_{n}} \\
& =\limsup _{n \rightarrow \infty} \sqrt[3 n+2]{\frac{4^{n}}{n^{3 / 2} \sqrt{\pi}}} \\
& =\sqrt[3]{4}
\end{aligned}
$$

Therefore, $B(0,1 / \sqrt[3]{4})$ is the disk of convergence of $h_{\infty}^{(2)}(\lambda)$ (observe that $r=1 / \sqrt[3]{4} \approx 0.6299$ ).

\section{Auxiliary Results}

Before proceeding further, we shall present some useful tools related to the previous sequences.

Our the first ingredient provides a useful form to the Laurent series of $h_{n}(\lambda)$.

Lemma 1. For any $n \geq 1$, there exists a power series $P_{n}(\lambda)$ such that

$$
h_{n}(\lambda)= \begin{cases}\lambda^{2}+\lambda^{5} P_{n}\left(\lambda^{3}\right), & \text { if } n \text { is even; } \\ \frac{1}{\lambda}+\lambda^{2} P_{n}\left(\lambda^{3}\right), & \text { if } n \text { is odd. }\end{cases}
$$

Proof. By definition in (1), $h_{n}(\lambda)$ satisfies the following recurrence relation

$$
h_{n+1}(\lambda)=\frac{1}{\left(h_{n}(\lambda)\right)^{2}+\lambda}
$$

with $h_{0}(\lambda)=0$ (since $\left.h_{1}(\lambda)=H(0, \lambda)=1 / \lambda\right)$. Now, by defining $f_{n}(\lambda):=\lambda h_{n}(\lambda)$ and using the previous recurrence, we obtain

$$
f_{n+1}(\lambda) / \lambda=\frac{1}{\left(f_{n}(\lambda) / \lambda\right)^{2}+\lambda}
$$

and so

$$
f_{n+1}(\lambda)=\frac{\lambda^{3}}{\left(f_{n}(\lambda)\right)^{2}+\lambda^{3}}
$$

with $f_{0}(\lambda)=0$. We claim that $f_{n}(\lambda)=g_{n}\left(\lambda^{3}\right)$ for some rational function $g_{n}(\lambda)$, where $n$ is any positive integer. Indeed, we can proceed by induction on $n$. For $n=1$, we can take $g_{1}(\lambda)=1$. Suppose (by induction hypothesis) that $f_{n}(\lambda)=g_{n}\left(\lambda^{3}\right)$, for some formal power series $g_{n}(\lambda)$, then, by (3), we have

$$
f_{n+1}(\lambda)=\frac{\lambda^{3}}{\left(g_{n}\left(\lambda^{3}\right)\right)^{2}+\lambda^{3}}=g_{n+1}\left(\lambda^{3}\right),
$$


where $g_{n+1}(\lambda)$ can be chosen by satisfying the recurrence

$$
g_{n+1}(\lambda)=\frac{\lambda}{\left(g_{n}(\lambda)\right)^{2}+\lambda}
$$

with $g_{0}(\lambda)=0$. The inductive process is finished. Observe that, since $h_{n}(\lambda)=\lambda^{-1} g_{n}\left(\lambda^{3}\right)$, then it suffices to prove that

$$
g_{n}(\lambda)= \begin{cases}\lambda+O\left(\lambda^{2}\right), & \text { if } n \text { is even; } \\ 1+O(\lambda), & \text { if } n \text { is odd }\end{cases}
$$

The proof is also by induction on $n$ (more precisely, a double induction). For the basis cases, we have $g_{1}(\lambda)=1=1+O(\lambda)$ and

$$
g_{2}(\lambda)=\frac{\lambda}{1+\lambda}=\lambda\left(1-\lambda+\lambda^{2}-\cdots\right)=\lambda+O\left(\lambda^{2}\right)
$$

where we used that for $|\lambda|<1$, one has $(1+\lambda)^{-1}=\sum_{k \geq 0}(-\lambda)^{n}$ (in general, it holds that $\left.(1+O(1))^{-1}=1+O(1)\right)$. Suppose that (4) is valid for all $n \in[1,2 k]$. Then,

$$
\begin{aligned}
g_{2 k+1}(\lambda) & =\frac{\lambda}{\left(g_{2 k}(\lambda)\right)^{2}+\lambda} \\
& =\frac{\lambda}{\left(\lambda+O\left(\lambda^{2}\right)\right)^{2}+\lambda} \\
& =\frac{\lambda}{\lambda+O\left(\lambda^{2}\right)} \\
& =\frac{1}{1+O(\lambda)}=1+O(\lambda),
\end{aligned}
$$

where we used $O\left(\lambda^{r}\right)+O\left(\lambda^{s}\right)=O\left(\lambda^{\min \{r, s\}}\right)$, since $|\lambda|<1$.

Now, we use the previous fact

$$
\begin{aligned}
g_{2 k+2}(\lambda) & =\frac{\lambda}{\left(g_{2 k+1}(\lambda)\right)^{2}+\lambda} \\
& =\frac{\lambda}{(1+O(\lambda))^{2}+\lambda} \\
& =\frac{\lambda}{1+O(\lambda)} \\
& =\lambda(1+O(\lambda))=\lambda+O\left(\lambda^{2}\right) .
\end{aligned}
$$

This completes the induction proof of (4).

Therefore, since $|\lambda|<1$, we can write

$$
g_{n}(\lambda)= \begin{cases}\lambda+\sum_{i \geq 2} c_{i} \lambda^{i}, & \text { if } n \text { is even; } \\ 1+\sum_{i \geq 1} c_{i} \lambda^{i}, & \text { if } n \text { is odd }\end{cases}
$$

and so

$$
h_{n}(\lambda)=\frac{1}{\lambda} g_{n}\left(\lambda^{3}\right)= \begin{cases}\lambda^{2}+\sum_{i \geq 2} c_{i} \lambda^{3 i-1}, & \text { if } n \text { is even; } \\ \frac{1}{\lambda}+\sum_{i \geq 1} c_{i} \lambda^{3 i-1}, & \text { if } n \text { is odd. }\end{cases}
$$

This completes the proof. 
Remark 2. Note that, by using Lemma 1, we can write

$$
h_{n}(\lambda)=\alpha_{-1, n} \lambda^{-1}+\alpha_{0, n} \lambda^{2}+\alpha_{1, n} \lambda^{5}+\cdots=\sum_{k=-1}^{\infty} \alpha_{k, n} \lambda^{3 k+2} \in \mathbb{R}[[\lambda]],
$$

where $\alpha_{-1, n}=\left(1-(-1)^{n}\right) / 2$, i.e., $\alpha_{-1, n}$ is 1 if $n$ is odd and 0 if $n$ is even. In particular, $h_{n}(\lambda)$ is an analytic function in some neighborhood of $\lambda=0$, when $n$ is even, and for $n$ odd, $h_{n}(\lambda)$ has a simple pole at origin (with residue equal to 1 ).

Remark 3. Another viewpoint of Lemma 1 (and consequently, of Remark 2) is that the $k$-th derivative of $h_{n}(\lambda)=0$ as $\lambda \rightarrow 0$, for any $k \equiv 0$ or $1(\bmod 3)$. This fact can also be proved by a harder (but maybe theoretically useful) combination of induction, the generalized Chain Rule (Faà di Bruno's formula) and the fact that all odd order derivatives of $H_{\lambda}(z):=H(z, \lambda)$ vanish (for fixed $\lambda$ ) at $z=0$. This last assertion follows from Cauchy's integral formula. Indeed, we have

$$
H_{\lambda}^{(2 n+1)}(0)=\frac{(2 n+1) !}{2 \pi i} \int_{\gamma_{R}} \frac{H_{\lambda}(\omega)}{\omega^{2 n+2}} d \omega=\frac{(2 n+1) !}{2 \pi i} \int_{\gamma_{R}} \frac{1}{\left(\omega^{2}+\lambda\right) \omega^{2 n+2}} d \omega,
$$

where $\gamma_{R}$ is the circle $\gamma(t):=R e^{i t}$, for $t \in[0,2 \pi]$ and $0<R<|\lambda|$. Now, we can use the partial fraction decomposition to deduce that

$$
\frac{1}{\left(\omega^{2}+\lambda\right) \omega^{2 n+2}}=\frac{A}{\omega+\sqrt{|\lambda|}}-\frac{A}{\omega-\sqrt{|\lambda|}}+\frac{B}{\omega^{2 n+2}},
$$

for computable constants $A$ and $B$. Hence, again by the Cauchy integral formula, we have

$$
\int_{\gamma_{R}} \frac{1}{\left(\omega^{2}+\lambda\right) \omega^{2 n+2}} d \omega=2 A \pi i f(0)-2 A \pi i f(0)+2 B \pi i f^{(2 n+1)}(0),
$$

where $f(z)=1$, for all $z$. Thus, $H_{\lambda}^{(2 n+1)}(0)$ is equal to zero as claimed.

Now we show the important connection of the sequence $\left(\alpha_{k, n}\right)_{k \geq 0}$ to the Catalan numbers. For the simplicity of notation, we use the following notation in the rest of the text:

$$
\alpha_{k, n}= \begin{cases}d_{k}, & \text { for odd } n \\ e_{k}, & \text { for even } n\end{cases}
$$

Lemma 2. Let $\left(C_{k}\right)_{k \geq 0}$ be the Catalan sequence. We have

(i) If $\left(d_{k}\right)_{k \geq 0}$ is defined by the recurrence,

$$
d_{k+1}=-\left(C_{1} d_{k}+\cdots+C_{k+1} d_{0}\right)-C_{k+2}
$$

with $d_{0}=-C_{0}$, then $d_{k}=-C_{k}$, for all $k \geq 0$.

(ii) If $\left(e_{k}\right)_{k \geq 1}$ is defined by the recurrence,

$$
e_{k+1}=C_{0} e_{k}+\cdots+C_{k-1} e_{1}+C_{k}
$$

with $e_{1}=C_{1}$, then $e_{k}=C_{k}$, for all $k \geq 1$.

Proof. Let us recall that Catalan numbers satisfy the Segner recurrence relation (see, e.g., [19], p. 117)

$$
C_{i+1}=\sum_{j=0}^{n} C_{j} C_{i-j}
$$

with $C_{0}=1$. 
(i). We shall proceed by induction on $k$. For $k=0$, one has $d_{0}=-C_{0}$ (by definition). Suppose $d_{t}=C_{t}$, for all $t \in[0, k]$. Then,

$$
\begin{aligned}
d_{k+1}= & -\left(C_{1} d_{k}+\cdots+C_{k+1} d_{0}\right)-C_{k+2} \\
= & C_{1} C_{k}+\cdots+C_{k+1} C_{0}-C_{k+2} \\
= & \underbrace{C_{1} C_{k}+\cdots+C_{k+1} C_{0}}_{C_{k+2}-C_{k+1}}-C_{k+2} \\
& =-C_{k+1}
\end{aligned}
$$

which completes the proof (where we used (6)).

(ii). Again by induction on $k$, the basis case $e_{1}=C_{1}$ follows by definition. Assume now that $e_{t}=C_{t}$, for all $t \in[1, k]$. Then, by the recurrence for $\left(e_{k}\right)_{k}$ together with the induction hypothesis, we obtain

$$
\begin{aligned}
e_{k+1}= & C_{0} e_{k}+\cdots+C_{k-1} e_{1}+C_{k} \\
= & C_{0} C_{k}+\cdots+C_{k-1} C_{1}+C_{k} \\
= & \underbrace{C_{0} C_{k}+\cdots+C_{k-1} C_{1}}_{C_{k+1}-C_{k}}+C_{k} \\
& =C_{k+1}
\end{aligned}
$$

which finishes the proof (where we used again (6)).

The next lemma gives a helpful recurrence for $C_{n}$, depending on the parity of $n$. The proof follows by induction together with (6) (we leave the details to the readers).

Lemma 3. Let $\left(C_{n}\right)_{n \geq 0}$ be the Catalan sequence. Then,

$$
C_{2 n}=2 \sum_{j=1}^{n} C_{j-1} C_{2 n-j} \text { and } C_{2 n+1}=C_{n}^{2}+2 \sum_{j=1}^{n} C_{j-1} C_{2 n-j+1},
$$

for all $n \geq 0$ (with $C_{0}=1$ ).

Now, we are ready to deal with the proof.

\section{The Proof of the Theorem 1}

First, observe that (2) can be rewritten for any as

$$
h_{2 j}(\lambda)=C_{0} \lambda^{2}+\cdots+C_{j-1} \lambda^{3 j-1}+O\left(\lambda^{3 j+2}\right)
$$

and

$$
h_{2 j+1}(\lambda)=\frac{1}{\lambda}-\left(C_{0} \lambda^{2}+\cdots+C_{j-1} \lambda^{3 j-1}\right)+O\left(\lambda^{3 j+2}\right),
$$

where we adopt the convention that $C_{0} \lambda^{2}+\cdots+C_{j-1} \lambda^{3 j-1}=0$ for $j=0$.

Now, we want to prove the following fact:

Claim. It holds that

$$
\left(C_{0} \lambda^{2}+\cdots+C_{n-1} \lambda^{3 n-1}\right)^{2}=C_{1} \lambda^{4}+\cdots+C_{n} \lambda^{3 n+1}+O\left(\lambda^{3 n+2}\right),
$$

for a non-negative integer $n$.

Proof. The proof is by induction on $n$. The identity is true for $n=0$, since $C_{1}=C_{0}^{2}$. Suppose that (9) holds, then one has 


$$
\begin{aligned}
\left(C_{0} \lambda^{2}+\cdots+C_{n-1} \lambda^{3 n-1}+C_{n} \lambda^{3 n+2}\right)^{2}= & \left(C_{0} \lambda^{2}+\cdots+C_{n-1} \lambda^{3 n-1}\right)^{2} \\
& +2\left(C_{0} \lambda^{2}+\cdots+C_{n-1} \lambda^{3 n-1}\right) C_{n} \lambda^{3 n+2}+\left(C_{n} \lambda^{3 n+2}\right)^{2}
\end{aligned}
$$

Since we desire to evaluate the identity up to $O\left(\lambda^{3 n+5}\right)$, then

$$
2\left(C_{0} \lambda^{2}+\cdots+C_{n-1} \lambda^{3 n-1}\right) C_{n} \lambda^{3 n+2}+\left(C_{n} \lambda^{3 n+2}\right)^{2}=2 C_{0} C_{n} \lambda^{3 n+4}+O\left(\lambda^{3 n+7}\right) .
$$

On the other hand, in the induction hypothesis

$$
\left(C_{0} \lambda^{2}+\cdots+C_{n-1} \lambda^{3 n-1}\right)^{2}=C_{1} \lambda^{4}+\cdots+C_{n} \lambda^{3 n+1}+O\left(\lambda^{3 n+2}\right),
$$

the terms of order $\lambda^{3 n+4}$ were neglected (since we were interested in $O\left(\lambda^{3 n+2}\right)$ ). Thus, we can improve the previous identity by considering these terms (note that this procedure does not affect the induction hypothesis). Additionally, since the sum of two numbers, which are congruent to 2 modulo 3 , is congruent to 1 modulo 3 , there is no term of magnitude $\lambda^{3 n+3}$ in $\left(C_{0} \lambda^{2}+\cdots+C_{n-1} \lambda^{3 n-1}\right)^{2}$. Let us also suppose that $n$ is odd (the even case is carried out along the same lines). We then have

$$
\begin{aligned}
\left(C_{0} \lambda^{2}+\cdots+C_{n-1} \lambda^{3 n-1}\right)^{2}= & C_{1} \lambda^{4}+\cdots+C_{n} \lambda^{3 n+1} \\
& +2\left(C_{1} C_{n-1}+\cdots+C_{(n-1) / 2} C_{(n+1) / 2}\right) \lambda^{3 n+4}+O\left(\lambda^{3 n+5}\right) .
\end{aligned}
$$

Now, we combine (10) and (11) together with Lemma 3 to arrive at

$$
\begin{aligned}
\left(C_{0} \lambda^{2}+\cdots+C_{n-1} \lambda^{3 n-1}+C_{n} \lambda^{3 n+2}\right)^{2}= & \left(C_{0} \lambda^{2}+\cdots+C_{n-1} \lambda^{3 n-1}\right)^{2} \\
& +2\left(C_{0} \lambda^{2}+\cdots+C_{n-1} \lambda^{3 n-1}\right) C_{n} \lambda^{3 n+2}+\left(C_{n} \lambda^{3 n+2}\right)^{2} \\
= & C_{1} \lambda^{4}+\cdots+C_{n} \lambda^{3 n+1} \\
& +2\left(C_{1} C_{n-1}+\cdots+C_{(n-1) / 2} C_{(n+1) / 2}\right) \lambda^{3 n+4}+O\left(\lambda^{3 n+5}\right) \\
& +2 C_{0} C_{n} \lambda^{3 n+4}+O\left(\lambda^{3 n+7}\right) \\
= & C_{1} \lambda^{4}+\cdots+C_{n+1} \lambda^{3 n+4}+O\left(\lambda^{3 n+5}\right)
\end{aligned}
$$

which finishes the proof of the claim.

Now, we return to the proof of (2). Again, the proof is by induction on $n$. For the basis case, we have

$$
h_{1}(\lambda)=\frac{1}{\lambda}
$$

and, by Lemma 1,

$$
h_{2}(\lambda)=\lambda^{2}+O\left(\lambda^{5}\right) .
$$

Suppose that (2) is true for $h_{n}(\lambda)$ with $n \in[1,2 j]$. Then, by the recurrence relation for $\left(h_{n}(\lambda)\right)_{n}$ together with the induction hypothesis, we infer that

$$
h_{2 j+1}(\lambda)=\frac{1}{\left(h_{2 j}(\lambda)\right)^{2}+\lambda}=\frac{1}{\left(C_{0} \lambda^{2}+\cdots+C_{j-1} \lambda^{3 j-1}+O\left(\lambda^{3 j+2}\right)\right)^{2}+\lambda} .
$$

However, we can use (9) to write

$$
\left(C_{0} \lambda^{2}+\cdots+C_{j-1} \lambda^{3 j-1}+O\left(\lambda^{3 j+2}\right)\right)^{2}+\lambda=C_{0} \lambda+C_{1} \lambda^{4}+\cdots+C_{j} \lambda^{3 j+1}+O\left(\lambda^{3 j+2}\right) .
$$

From Lemma 1 and Remark 2, one has

$$
h_{2 j+1}(\lambda)=\frac{1}{\lambda}+d_{0} \lambda^{2}+d_{1} \lambda^{5}+\cdots+d_{j-1} \lambda^{3 j-1}+O\left(\lambda^{3 j+2}\right) .
$$


Thus, the coefficients $d_{0}, d_{1}, \ldots, d_{j-1}$ satisfy the following equality

$$
\begin{aligned}
1 \equiv\left(C_{0} \lambda+C_{1} \lambda^{4}+\cdots\right. & \left.+C_{k} \lambda^{3 j+1}+O\left(\lambda^{3 j+2}\right)\right)\left(\frac{1}{\lambda}+d_{0} \lambda^{2}+d_{1} \lambda^{5}+\cdots+d_{j-1} \lambda^{3 j-1}+O\left(\lambda^{3 j+2}\right)\right) \\
& \text { and so } \\
1 & \equiv 1+\lambda\left(\sum_{i=0}^{j-1} d_{i} \lambda^{3 i+2}\right)+C_{1} \lambda^{4}\left(\frac{1}{\lambda}+\sum_{i=0}^{j-2} d_{i} \lambda^{3 i+2}\right)+\cdots+C_{k} \lambda^{3 j+1}\left(\frac{1}{\lambda}\right)+O\left(\lambda^{3 j+1}\right) .
\end{aligned}
$$

By reordering this sum, we obtain

$$
0 \equiv\left(d_{0}+C_{1}\right) \lambda^{3}+\left(d_{1}+C_{1} d_{0}+C_{2}\right) \lambda^{6}+\cdots+\left(d_{j-1}+C_{1} d_{j-2}+\cdots+C_{j}\right) \lambda^{3 j}+O\left(\lambda^{3 j+1}\right) .
$$

Therefore, $d_{0}=-C_{1}=-C_{0}$ and

$$
d_{t}=-\left(C_{1} d_{t-1}+\cdots+C_{t} d_{0}\right)-C_{t}
$$

for all $t \in[1, j-1]$. By Lemma 3 (i), we conclude that $d_{t}=-C_{t}$, for all $t \in[1, j-1]$ which yields that

$$
h_{2 j+1}(\lambda)=\frac{1}{\lambda}-\left(C_{0} \lambda^{2}+C_{1} \lambda^{5}+\cdots+C_{k-1} \lambda^{3 j-1}\right)+O\left(\lambda^{3 j+2}\right)
$$

as desired.

Thus, we determine that (2) holds for $h_{n}(\lambda)$ for all $n \in[1,2 j+1]$. To finish the proof, we must prove that (2) is also true for $n=2 j+2$. First, one has that

$$
h_{2 j+2}(\lambda)=\frac{1}{\left(h_{2 j+1}(\lambda)\right)^{2}+\lambda}=\frac{1}{\left((1 / \lambda)-\left(C_{0} \lambda^{2} \cdots+C_{j-1} \lambda^{3 j-1}\right)+O\left(\lambda^{3 j+2}\right)\right)^{2}+\lambda} .
$$

However, by (9) and after a straightforward calculation, we arrive at

$$
\left(h_{2 j+1}(\lambda)\right)^{2}+\lambda=\frac{1}{\lambda^{2}}-\left(C_{0} \lambda+C_{1} \lambda^{4}+\cdots+C_{j-1} \lambda^{3 j-2}\right)+C_{k} \lambda^{3 j+1}+O\left(\lambda^{3 j+2}\right) .
$$

Now, we use Lemma 1 (and Remark 2) to write

$$
h_{2 j+2}(\lambda)=e_{0} \lambda^{2}+e_{1} \lambda^{5}+\cdots+e_{j} \lambda^{3 j+2}+O\left(\lambda^{3 j+5}\right),
$$

where $e_{0}=1$. Hence,

$$
1 \equiv\left(\frac{1}{\lambda^{2}}-\left(C_{0} \lambda+\cdots+C_{j-1} \lambda^{3 j-2}\right)+C_{k} \lambda^{3 j+1}+O\left(\lambda^{3 j+2}\right)\right)\left(\lambda^{2}+e_{1} \lambda^{5}+\cdots+e_{j} \lambda^{3 j+2}+O\left(\lambda^{3 j+5}\right)\right) .
$$

Thus,

$$
1 \equiv 1+\lambda^{-2}\left(\sum_{i=1}^{j} e_{i} \lambda^{3 i+2}\right)-C_{0} \lambda\left(\sum_{i=0}^{j} e_{i} \lambda^{3 i+2}\right)+\cdots+C_{j-1} \lambda^{3 j-2} \cdot \lambda^{2}+O\left(\lambda^{3 j+3}\right)
$$

which can be re-written as

$$
0 \equiv\left(e_{1}-1\right) \lambda^{3}+\left(e_{2}-C_{0} e_{1}-C_{1}\right) \lambda^{6}+\cdots+\left(e_{j}-e_{j-1}-C_{1} e_{j-2}-\cdots-C_{j-2} e_{1}+C_{j-1}\right) \lambda^{3 j}+O\left(\lambda^{3 j+3}\right) .
$$

We then deduce that $e_{1}=1=C_{1}$ and

$$
e_{t}=C_{0} e_{j-1}+C_{1} e_{j-2}+\cdots+C_{j-2} e_{1}-C_{j-1}
$$


for all $t \in[1, j]$. By Lemma 3 (ii), we have $e_{t}=C_{t}$, for all $t \in[1, j]$, yielding that

$$
h_{2 j+2}(\lambda)=C_{0} \lambda^{2}+C_{1} \lambda^{5}+\cdots+C_{j} \lambda^{3 j+2}+O\left(\lambda^{3 j+5}\right) .
$$

The proof is then complete.

\section{Conclusions}

This paper is devoted to the proof of a conjecture formulated by Mork and Ulness ([16], Conjecture 4.2). Roughly speaking, they computationally observed the relation between the coefficients of $h_{n}(\lambda)$ (the $n$-th iteration of $1 /\left(z^{2}+\lambda\right)$ at $\left.z=0\right)$ and the Catalan sequence $\left(C_{k}\right)_{k}$. Indeed, we prove a quantitative version of their conjecture by showing that the sequence $\left(h_{n}(\lambda)-\left(\frac{1-(-1)^{n}}{2 \lambda}+(-1)^{n} \sum_{i=1}^{\lfloor n / 2\rfloor} C_{i-1} \lambda^{3 i-1}\right)\right)_{n}$ tends to zero (with order $\left.|\lambda|^{3\lfloor n / 2\rfloor+2}\right)$ as $n \rightarrow \infty$.

Author Contributions: P.T. and K.V. conceived of and designed the investigation and provided background for the investigation; P.T. applied the Mathematica code to perform the investigation; both authors analyzed the data; K.V. wrote the original draft of manuscript; both authors edited the manuscript. Both authors have read and agreed to the published version of the manuscript.

Funding: The authors was supported by the Project of Specific Research PrF UHK no. 2101/2021, University of Hradec Králové, Czech Republic.

Institutional Review Board Statement: Not applicable.

Informed Consent Statement: Not applicable.

Data Availability Statement: Not applicable.

Acknowledgments: The authors would like to thank anonymous referees for their careful corrections and their comments that helped to improve the quality of the paper.

Conflicts of Interest: The author declares no conflict of interest.

\section{References}

1. Yang, G. Some Geometric Properties of Julia Sets and filled-in Julia Sets of Polynomials. Complex Var. Theory Appl. 2002, 47, 383-391. [CrossRef]

2. Falconer, K.J. Fractal Geometry: Mathematical Foundations and Applications, 2nd ed.; John Wiley and Sons: Chichester, UK, 2003.

3. Berry, M.V.; Lewis, Z.V.; Nye, J.F. On the Weierstrass-Mandelbrot fractal function. Proc. R. Soc. Lond. 1980, 370, 459-484. [CrossRef]

4. Guariglia, E. Primality, Fractality, and Image Analysis. Entropy 2019, 21, 304. [CrossRef] [PubMed]

5. Berteloot, F.; Dinh, T.C. The Mandelbrot set is the shadow of a Julia set. Discret. Contin. Dyn. Syst. 2020, 40, 6611-6633. [CrossRef]

6. Sun, T.; Wang, D. The Symmetry in the Noise-Perturbed Mandelbrot Set. Symmetry 2019, 11, 577. [CrossRef]

7. Blankers, V.; Rendfrey, T.; Shukert, A.; Shipman, P.D. Julia and Mandelbrot Sets for Dynamics over the Hyperbolic Numbers. Fractal Fract. 2019, 3, 6. [CrossRef]

8. Zhou, H.; Tanveer, M.; Li, J. Comparative study of some fixed-point methods in the generation of Julia and Mandelbrot sets. J. Math. 2020, 2020, 7020921. [CrossRef]

9. Abbas, M.; Iqbal, H.; De la Sen, M. Generation of Julia and Mandelbrot Sets via Fixed Points. Symmetry 2020, 12, 86. [CrossRef]

10. Tanveer, M.; Ahmed, I.; Raza, A.; Nawaz, S.; Lv, Y.P. New escape conditions with general complex polynomial for fractals via new fixed point iteration. AIMS Math. 2021, 6, 5563-5580. [CrossRef]

11. Mork, L.K.; Vogt, T.; Sullivan, K.; Rutherford, D.; Ulness, D.J. Exploration of Filled-In Julia Sets Arising from Centered Polygonal Lacunary Functions. Fractal Fract. 2019, 3, 42. [CrossRef]

12. Kahane, J.-P. Lacunary Taylor and Fourier series. Bull. Am. Math. Soc. 1964, 70, 199-213. [CrossRef]

13. Gaposhkin, V.F. Lacunary series and independent functions. Uspekhi Matematicheskikh Nauk 1966, 21, 3-82. [CrossRef]

14. Hille, E. Analytic Function Theory; Ginn and Company: Boston, MA, USA, 1959; Volume I.

15. Mork, L.K.; Sullivan, K.; Ulness, D.J. Lacunary Möbius Fractals on the Unit Disk. Symmetry 2021, 13, 91. [CrossRef]

16. Mork, L.K.; Ulness, D.J. Visualization of Mandelbrot and Julia Sets of Möbius Transformations. Fractal Fract. 2021, 5, 73. [CrossRef]

17. Wolfram, S. The Mathematica Book, 4th ed.; Wolfram Media/Cambridge University Press: Cambridge, UK, 1999.

18. Sloane, N.J.A. The On-Line Encyclopedia of Integer Sequences. Available online: https: / / oeis.org/ (accessed on 31 July 2021).

19. Koshy, T. Catalan Numbers with Applications; Oxford University Press: Oxford, UK, 2009.

20. Dutton, R.D.; Brigham, R.C. Computationally Efficient Bounds for the Catalan Numbers. Eur. J. Comb. 1986, 7, 211-213. [CrossRef] 